\title{
Resonant Transmission through Slits in a Cavity inside a Thin Conducting Plane
}

\author{
Jong-Ig Lee ${ }^{1} \cdot$ Young-Ki Cho ${ }^{2}$
}

\begin{abstract}
In this paper, the problem of electromagnetic transmission via slits in a cavity inside a conducting screen of finite thickness has been considered in the case that the transverse electric(to the slit axis) polarized plane wave is incident on a slit. The problem is solved numerically by the method of moments and the results are compared with those obtained from an equivalent circuit suitable for a case in which the slit width is infinite and the structure is modified to the two partially overlapped conducting planes. It is observed that when the cavity is resonated, the effective slit width reaches its maximum value of $1 / \pi$ wavelengths, irrespective of the actual slit width and the incidence angle. When the thickness of the conducting plane is much smaller than the wavelength, the numerical results for the effective slit width(or transmission width) agree well with those obtained from the equivalent circuit, even though the slit is as narrow as the thickness of the conducting plane.
\end{abstract}

Key words: Resonant Transmission, Narrow Slit, Effective Slit Width, Cavity Resonance.

\section{Introduction}

An electromagnetic problem of considerable academic and practical importance is that of determining the electromagnetic energy coupled(or transmitted) from one region to another through transmission-cavity resonance(TCR) structures ${ }^{[1] \sim[5]}$. The most representative structure for this study is the aperture-cavity-aperture system ${ }^{[1]}$, where the structure uses the input/output transverse walls containing small coupling holes to form the transmission resonance cavity.

In contrast to this type, a distinctive type of transmission resonance cavity is constituted simply by the slot in a thick conducting screen as in [2], [3]. This type of transmission resonance structure has been closely studied in the hope that the transmission resonance mechanism via such a structure might explain the enhancement of transmission observed in recent experiments on an extraordinary optical transmission(EOT) problem through a twodimensional(2-D) array of sub-wavelength holes perforated in a thick metallic film ${ }^{[4],[5]}$.

Most studies $^{[1] \sim[5]}$ on these types of transmission resonance problems, however, have considered the resonant cavity structures formed in a thick conducting screen whose thickness corresponds to the cavity length. Hence, in this study, we have explored a transmission cavity structure in a thin conducting screen as shown in Fig. 1, in which two slits are introduced to the edges of the cavity inside a thin conducting plane to form a cavity region between the slits. The structure in Fig. 1 is illuminated by a TE(to the slit axis) polarized plane wave, and the problem of electromagnetic transmission is analyzed using the method of moments $(\mathrm{MoM})^{[6]}$.

In the case of a wide slit, in which the slit is so wide that the effect of diffraction from the slit edges can be neglected, the transmission cavity structure in Fig. 1 might be approximated to the parallel conducting planes partially overlapped as shown in Fig. 2. The results for the effective slit width obtained from the analysis of the problem in Fig. 1 is compared with those computed using an equivalent circuit ${ }^{[7],[8]}$ of Fig. 3 for the simplified structure(Fig. 2), in order to show that, in the case of a thin conducting plane, the power transmission through the cavity between the slits is not affected significantly by the diffraction from the slit edge even if the slit width is as small as the conductor thickness. In addition, the results show that although the cavity structure formed in a thin conducting plane (Fig. 1) is a structure seemingly different to that in the thick conducting screen in the previous structure ${ }^{[2]}$, the transmission resonance phenomena are similar in essence.

\footnotetext{
Manuscript received April 14, 2010 ; revised August 20, 2010. (ID No. 20100414-02J)

${ }^{1}$ Department of Electronics Engineering, Dongseo University, Busan, Korea.

${ }^{2}$ School of Electrical Engineering and Computer Science, Kyungpook National University, Daegu, Korea.

Corresponding Author : Jong-Ig Lee (e-mail : leeji@gdsu.dongseo.ac.kr)
} 


\section{Analysis}

Fig. 1 illustrates the structure of the transmission cavity formed by two slits at the edges of the cavity region 3 inside the conducting plane of thickness $d$. In Fig. 1, the thickness $d$ of the conducting plane is small compared to the wavelength $\lambda_{0}$ and the slit width $w$ is assumed to be greater than the conductor thickness $d$. The transmission cavity ranges between the inner edges of the slits, i.e., $0 \leq x \leq d, 0 \leq y \leq L$, and so $L$ is the cavity length and $d$ is the gap height. The TE(to the slit axis) polarized plane wave $\left(E_{z}=0\right)$ is incident on the structure with an incidence angle of $\phi_{0}$.

The transmission resonance(or maximum power transmission) in this structure can be expected when the cavity length $L$ approaches near the multiples of the half wavelengths. This resonance is in contrast to the previous cavity structures ${ }^{[1] \sim[5]}$ of the small apertures in a thick conducting plane, which show a transmission resonance when its thickness becomes close to the half wavelengths. The medium constants for each region are assumed to be $\left(\mu_{0}, \varepsilon_{0}\right)$ of the free space, but they have no limitation in the extension of this study to the case of dielectric material having an arbitrary constant $\varepsilon_{r}$.

The electromagnetic problem in Fig. 1 can be solved using the method of moments(MoM) as described in [6], and so a detailed analysis procedure is omitted here. From knowledge of the tangential electric field distributions $E_{y 1}(y)\left[E_{y}(0, y)\right]$ and $E_{y 2}(y)\left[E_{y}(d, y)\right]$ over slits 1 and 2 , one can compute the coupled power $P_{s 2}[\mathrm{~W} / \mathrm{m}]$, per unit length along the $z$ axis, into the region 2. The effective slit width $w_{\text {eff }}$ of the transmission cavity structure in Fig. 1 can be obtained as the ratio of the transmithed power $P_{s 2}[\mathrm{~W} / \mathrm{m}]$ to the incident power density $P_{i}\left(=0.5 \eta_{0} H_{0}^{2}\left[\mathrm{~W} / \mathrm{m}^{2}\right]\right)$, i.e.,

$$
w_{e f f}=P_{s 2} / P_{i}[\mathrm{~m}] .
$$

If the slit width $w$ in Fig. 1 is increased to a sufficiently large value such as the diffraction effect of the

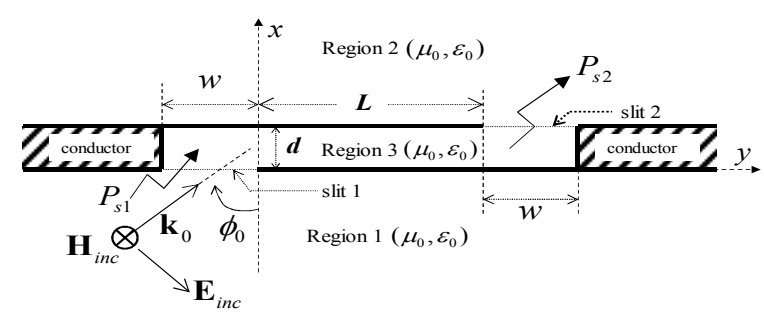

Fig. 1. Proposed transmission cavity structure.

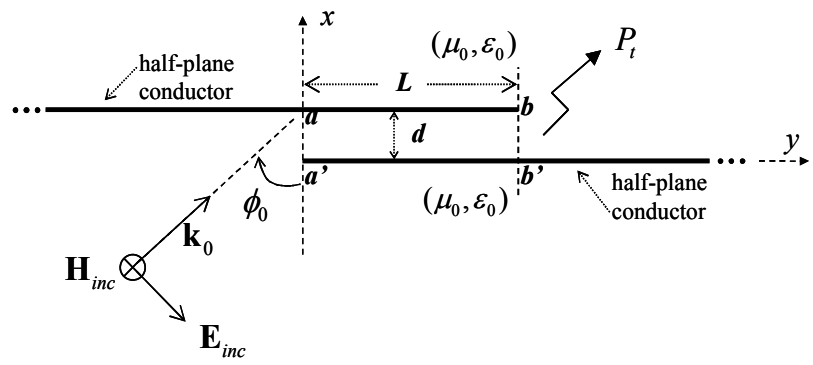

Fig. 2. Partially overlapped parallel conducting planes.

conducting right angle edges at $y=-w$ and $y=L+w$ can be ignored, then the structure might be simplified to that shown in Fig. 2. Fig. 2 shows another structure of the transmission cavity, which is composed of two partially overlapped parallel conducting planes with a small gap distance $d$ and overlapped length $L$.

The equivalent circuit for the structure of Fig. 2 is given in Fig. 3, which is of the same form as that of the narrow slit problem in the thick conducting screen ${ }^{[2]}$. In this equivalent circuit, $Y_{r}(=G+j B)$ represents the radiation admittance for both ports $a-a^{\prime}$ and $b-b^{\prime}$, which can be obtained through the use of the method in [6]. When the distance $d$ between two conducting plates is small(i.e., $\left.k_{0} d \ll 1\right)$, the radiation conductance $G(=1 /(120$ $\left.\left.\lambda_{0}\right)[\mathrm{S} / \mathrm{m}]\right)$ in Fig. 3 is identical to that of the narrow slit in a flanged parallel-plate waveguide ${ }^{[2]}$, as shown in [7], [8].

By using the above equivalent circuit representation, the transmission resonance condition is obtained and summarized as follows:

At resonance, the total input admittance at port $a-a^{\prime}$ becomes purely real as $Y_{i n}^{a-a^{\prime}}=2 G[\mathrm{~S} / \mathrm{m}]$ and the resonant length $L_{r e s}$ of the cavity is approximately given by [2], [3]

$$
L_{\text {res }} \cong\left(n-\frac{2 B}{\pi Y_{0}}\right) \frac{\lambda_{0}}{2}, n=1,2,3, \cdots
$$

with the assumption that $G \ll B$ and $|G+j B|<<Y_{0}$ for $k_{0} d$ $\ll 1$. Under this resonance condition, the power trans-

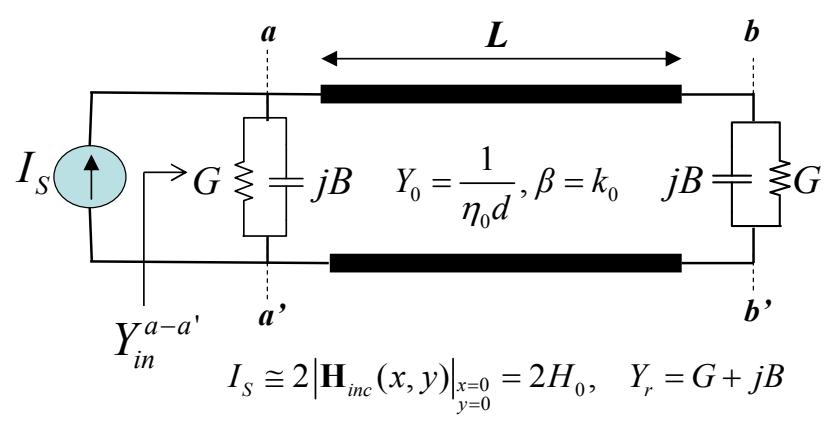

Fig. 3. Equivalent circuit for the problem in Fig. 2. 
ferred to the load at port $b-b^{\prime}$ becomes $P_{G}^{b-b^{\prime}}=H_{0}^{2} /$ $2 G[\mathrm{~W} / \mathrm{m}]$, which corresponds to the power $P_{t}$, per unit length along the $z$ axis, transmitted through the gap region $(0<x<d)$, and reradiated into the upper half space $(x>0)$ in Fig. 2.

If we define the effective gap height $d_{\text {eff }}$ as the ratio of the transmitted power $P_{t}\left(=P_{G}^{b-b^{\prime}}\right)[\mathrm{W} / \mathrm{m}]$ to the incident power density $P_{i}$, i.e.,

$$
d_{e f f}=P_{t} / P_{i}[\mathrm{~m}],
$$

the maximum effective gap height is found to be $\lambda_{0} / \pi$ $[\mathrm{m}]$ independent of the incidence angle $\phi_{0}$ and the actual gap height $d$. This result of $\lambda_{0} / \pi[\mathrm{m}]$ is the same as the well-known effective height of the half-wavelength dipole antenna as well as that of the transmission width determined in a previous study ${ }^{[2]}$.

\section{Numerical Results and Discussions}

In order to examine the validity of our numerical results, we checked the power conservation relation $P_{s 1}$ $=P_{s 2}=P_{2 \text { rad }}$, where $P_{s 1}\left(P_{s 2}\right)$ is the coupled power through slit 1(slit 2) and $P_{2 \text { rad }}$ is the radiated power into the upper half space region 2 , and the relative error remained to be less than $10^{-4}$. As another method of validation, we compared our results for the magnetic current distributions over the slits with those of Fig. 8 in [6]. In addition, as will be shown later in the explanation of Figs. 4 and 5, the results obtained by the MoM for the effective slit width $w_{\text {eff }}$ of the structure in Fig. 1 correspond well with the effective gap height $d_{\text {eff }}$ obtained through the use of the equivalent circuit approach.

For the sake of investigating the effect of the right angle edge at $y=-w($ or $y=L+w)$ on the transmission through the slit edge at $y=0$ ( or $y=L$ ), we have calculated the effective slit width $w_{\text {eff }}$ for three cases where $w=d, 2 d$, and $5 d$ with $d$ kept constant as $d=0.01 \lambda_{0}$ and compared these results with the effective gap height $d_{\text {eff }}$ obtained through the use of the equivalent circuit approach, as shown in Fig. 4(a) (in this case of $d=0.01 \lambda_{0}$, the equivalent admittance $Y_{r}$ obtained through the use of the method in [7], [8] is $Y_{r}=0.008+j 0.027[\mathrm{~S} / \mathrm{m}]$ when the guide height $b=0.01 \lambda_{0}$ and the slit width $2 a=0.05 \lambda_{0}=5 b$ in Fig. 1 in [7]). The results show that the curves are virtually identical in all three cases, independent of the slit width $w$, and are also in accordance with the results for $d_{\text {eff }}$ obtained through the use of the equivalent circuit. Therefore, when $d$ is very small in comparison with $\lambda_{0}$, the power transmitted through the cavity region and reradiated into the upper half space in Fig. 2 becomes virtually identical to that in Fig. 1, if the slit width $w$ is chosen to be at least larger than $d$.

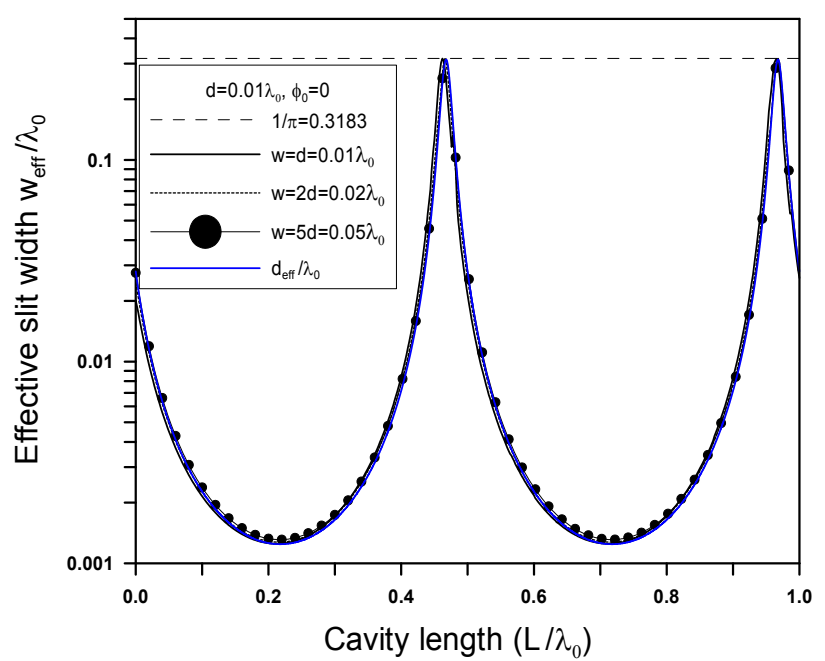

(a) Effect of slit width $w\left(\phi_{0}=0\right)$

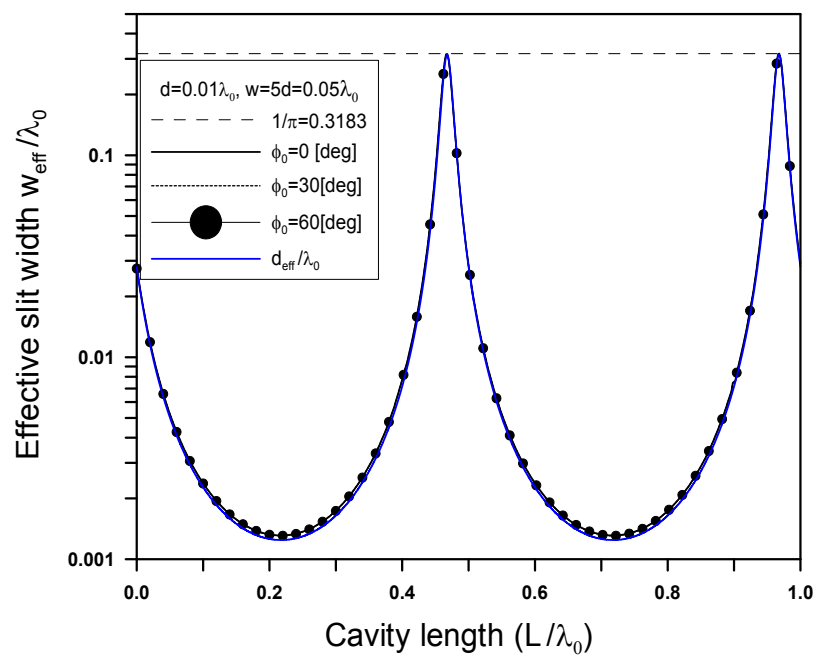

(b) Effect of incidence angle $\Phi_{0}\left(w=5 d=0.05 \lambda_{0}\right)$

Fig. 4. Effective slit width $w_{\text {eff }}$ vs. cavity length $L\left(d=0.01 \lambda_{0}\right)$.

It can be seen that the maximum value of the transmission width becomes $\lambda_{0} / \pi[\mathrm{m}]$ in Fig. 4(a), as expected. In Fig. 4(a), resonant cavity length $L_{\text {res }}$ calculated by the equivalent circuit approach is $0.4677 \lambda_{0}$, which is very close to $0.4676 \lambda_{0}$ predicted by (1) and $0.467 \lambda_{0}$ in the curve for $w_{s}=5 d$.

Next, in order to check the dependence of the results on the incidence angle $\phi_{0}$, the incidence angle is changed from 0 to 30 and 60 degrees with the slit width fixed $(w=5 d)$; the results are compared with each other in Fig. 4(b). As shown in Fig. 4(b), in the case of small thickness $d=0.01 \lambda_{0}$, the characteristics of the power transmission do not change appreciably against the incidence angle $\phi_{0}$.

Fig. 5 shows the effects of the slit width $w$ and the incidence angle $\phi_{0}$ when the thickness of the conducting plane is increased to $d=0.02 \lambda_{0}$. Unlike the former 


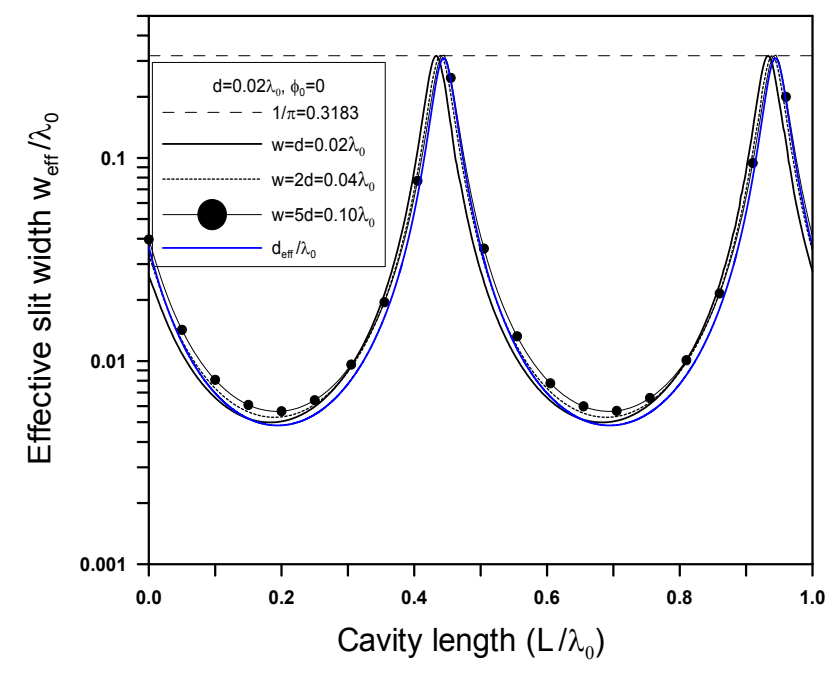

(a) Effect of slit width $w\left(\phi_{0}=0\right)$

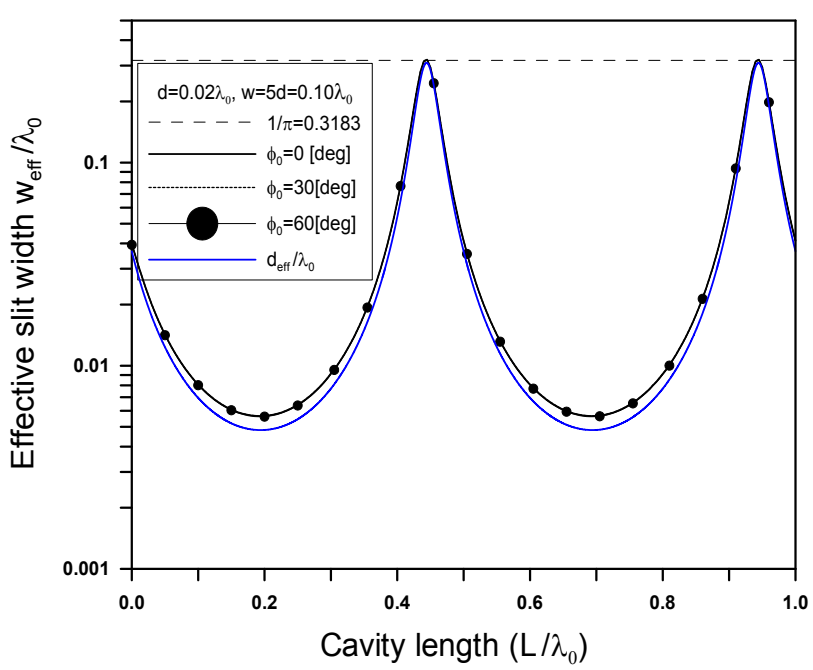

(b) Effect of incidence angle $\Phi_{0}\left(w=5 d=0.10 \lambda_{0}\right)$

Fig. 5. Effective slit width $w_{\text {eff }}$ vs. cavity length $L\left(d=0.02 \lambda_{0}\right)$.

case of $d=0.01 \lambda_{0}$, the power transmission begins to be affected by the geometrical parameters(especially slit width $w$ rather than incidence angle $\phi_{0}$ ), which result from the influence of the diffraction from the right angle edges. Fig. 5(a) shows that when the actual slit width $w$ in Fig. 1 is increased, the curves for the effective slit width $w_{\text {eff }}$ converge to the curve for the effective gap height $d_{\text {eff }}$ of the structure in Fig. 2, which is thought to be an extreme case of the structure in Fig. 1(i.e., the case of infinite slit width, $w=\infty)$.

From the enlarged views of Fig. 4(a) and Fig. 5(a), it is observed that, as the slit width $w$ in Fig. 1 is increased, the resonant cavity length where $w_{\text {eff }}$ is maximized is increased. This increase might be due to the decrease in the stored reactive power near the widened slit region as can be predicted from (2).

\section{Conclusions}

The problem of electromagnetic resonant transmission through slits in a cavity embedded inside a thin conducting plane is studied when the slit is illuminated by a $\mathrm{TE}$ (to the slit axis) polarized plane wave. From the results for the effective slit width, it is found that the maximum effective slit width amounts to $1 / \pi$ wavelengths, nearly irrespective of the slit width and the incidence angle of the plane wave, and the variations of the effective slit width corresponds well with those obtained using simple equivalent circuit representation for the case of infinite slit width.

This work was supported by Dongseo University Research Fund of 2009.

\section{References}

[1] Y. Leviatan, R. F. Harrington, and J. R. Mautz, "Electromagnetic transmission through apertures in a cavity in a thick conductor", IEEE Trans. Antennas Propagat., vol. AP-30, no. 6, pp. 1153-1165, Nov. 1982.

[2] R. F. Harrington, D. T. Auckland, "Electromagnetic transmission through narrow slots in thick conducting screens", IEEE Trans. Antennas Propagat., vol. AP-28, no. 5, pp. 616-622, Sep. 1980.

[3] Y. K. Cho, K. W. Kim, J. H. Ko, and J. I. Lee, "Transmission through a narrow slot in a thick conducting screen", IEEE Trans. Antennas Propagat., vol. AP-57, no. 3, pp. 813-816, Mar. 2009.

[4] Y. Takakura, "Optical resonance in a narrow slit in a thick metallic screen", Phys. Rev. Lett., vol. 86, no. 24, pp. 5601-5603, Jun. 2001.

[5] F. Yang, J. R. Sambles, "Resonant transmission of microwaves through a narrow metallic slit", Phys. Rev. Lett., vol. 89, no. 6, pp. 063901(1)-063901(3), Aug. 2002.

[6] D. T. Auckland, R. F. Harrington, "Electromagnetic transmission through a filled slit in a conducting plane of finite thickness, TE case", IEEE Trans. Microwave Theory Tech., vol. MTT-26, no. 7, pp. 499-505, Jul. 1978.

[7] Y. K. Cho, "On the equivalent circuit representation of the slitted parallel-plate waveguide filled with a dielectric", IEEE Trans. Antennas Propagat., vol. AP37, no. 9, pp. 1193-1200, Sep. 1989.

[8] J. I. Lee, J. H. Ko, and Y. K. Cho, "A note on diffraction and equivalent admittance properties of a transverse slit in a parallel-plate waveguide", IEICE Trans Com., vol. E86-B, pp. 3600-3605, 2003. 


\section{Jong-Ig Lee}

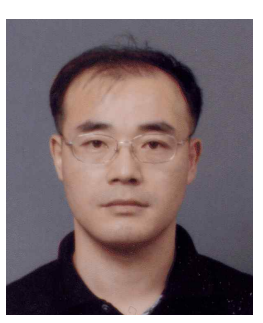

received the B.S., M.S., and Ph.D. degrees in electronics engineering from Kyungpook National University in 1992, 1994, and 1998, respectively. In 1998, he was a Research Professor at the School of Electronics, Kumoh National University of Technology, Gumi, Korea. Currently, he is with Dongseo University, Busan, Korea, as an associate professor in the Department of Electronics Engineering. Dr. Lee received the 1996 International Symposium on Antennas and Propagation('96ISAP) "Young Scientist Award.". His research interests include electromagnetic scattering and planar antenna theory.

\section{Young-Ki Cho}

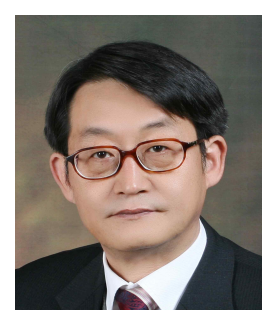

received a B.S. degree in electrical engineering from Seoul National University, Seoul, Republic of Korea, in 1978 and M.S. and Ph.D. degrees in electrical engineering from the Korea Advanced Institute of Science and Technology(KAIST), Daejeon, Korea, in 1981 and 1998, respectively. In October 1981, he joined the School of Electrical Engineering and Computer Science at Kyungpook National University, Daegu, Korea. Since 1992 he has been Korea's representative on URSI Commission B, Fields and Waves, and in 2008, he became President of the Korean Institute of Electromagnetic Engineering and Science(KIEES). His research interests are in the areas of electromagnetic scattering, microwaves, optics, and antenna theory. 